\title{
Towards Meson Spectroscopy Instead of Bump Hunting
}

\author{
George Rupp, ${ }^{1}$ Susana Coito, ${ }^{1}$ Eef van Beveren ${ }^{2}$ \\ ${ }^{1}$ Centro de Física das Interacções Fundamentais, Instituto Superior Técnico, \\ Technical University of Lisbon, P-1049-001 Lisboa, Portugal \\ ${ }^{2}$ Centro de Física Computacional, Departamento de Física, \\ Universidade de Coimbra, P-3004-516 Coimbra, Portugal
}

September 10, 2018

\begin{abstract}
Mesonic resonances are generally observed in data as narrow, moderately broad, or wide peaks in scattering or production processes. In the eyes of nearly all experimentalists, any suchlike bump is a true resonance as soon as its statistical significance exceeds certain minimal values. However, this simple point of view ignores possible effects from competing hadronic channels and the opening of the corresponding thresholds. On the other hand, most theoretical hadron-model builders consider mesons merely bound states of a quark and an antiquark, or of more exotic combinations sometimes involving valence gluons as well. Also the latter description is much too naive, since considerable mass shifts or even the dynamical generation of extra states due to unquenching are equally ignored.

In the present paper, a largely empirical yet very successful approach to meson spectroscopy is revisited, in which all the above phenomena can be accounted for non-perturbatively, with concrete examples of some enigmatic mesonic states described in detail. First, the $X(4260)$ charmonium enhancement is argued to be a non-resonant structure resulting from depletion effects due to competing channels and resonances. Then, the $X(3872)$ charmonium-like meson is described as a unitarised $J^{P C}=1^{++} c \bar{c}$ state. Also, the unusual pattern of masses and widths of the open-charm axial-vector mesons $D_{1}(2420), D_{1}(2430), D_{s 1}(2536)$, and $D_{s 1}(2460)$ is shown to follow from highly non-perturbative coupled-channel and mixing effects. Finally, first indications of a very light scalar boson are presented, on the basis of published BABAR data.
\end{abstract}

\section{Introduction}

Meson spectroscopy is an essential tool in trying to understand both the confinement and the decay mechanism in QCD. The large volume of experimental data [1] on mesonic bound states and resonances, from the light-quark sector all the way up to bottomonium, should allow to draw far-reaching and detailed conclusions on the nature of the confining potential and how it is affected by the possibility of strong decay, via $q \bar{q}$ pair creation. However, a necessary condition for a systematic analysis of mesonic data for spectroscopy purposes is the ability to distinguish between true resonances and enhancements that originate in threshold effects, as well as inelasticities due to competing channels and true resonances. Especially in charmonium, several intriguing structures, a couple of which even charged, have been 
observed [1] above the lowest open-charm threshold that may not be resonances at all [2]. Very recently, also in bottomonium two charged enhancements were reported [3], which happen to lie just a few $\mathrm{MeV}$ above the $B B^{*}$ and $B^{*} B^{*}$ thresholds, respectively. These may be other examples of non-resonant structures that are just threshold cusps [4]. However, see Ref. [5] for a different point of view.

On the other hand, there are true resonances, too, that do not show up as clear bumps in the data, or are not accompanied by a scattering phase shift rising past $90^{\circ}$. Examples of the latter case are the light scalar mesons [6, 7] $f_{0}(600)$ [1] (alias $\sigma$ ) and $K_{0}^{*}(800)$ (alias $\kappa$ ) [1]. What makes their experimental observation so difficult is a combination of factors, namely their very large widths, slowly rising phase shifts due do an Adler zero slightly below the lowest threshold (at $\approx m_{\pi}^{2} / 2$ for $\sigma, \approx m_{K}^{2}-m_{\pi}^{2} / 2$ for $\kappa$ ), and partly overlapping resonances with the same quantum numbers $\left(f_{0}(980)\right.$ for $\sigma, K_{0}^{*}(1430)$ for $\left.\kappa\right)$.

Thus, non-resonant enhancements and hard to observe resonances complicate the task of meson spectroscopists in trying to systematically describe the available data. However, an additional and often overlooked difficulty is the inevitable mass shift of $q \bar{q}$ states, bound by some confining potential, due to the possibility of quark-pair creation and annihilation, which gives rise to meson-loop contributions, even for states below all strong decay threshold. These shifts can, in principle, be as large as the hadronic decay widths of many mesons, i.e., of the order of hundreds of MeV. Moreover, in general one is dealing with complex and non-linear energy shifts, governed by analytic $S$-matrix properties, the widths being generated by the very same mechanism. In view of such effects, it is hard to understand why so many model builders immediately resort to exotic configurations, such as tetraquarks and hybrids, whenever a newly observed mesonic resonance does not seem to fit in the usual quark potential models. Rather, one should try to "unquench" these models, in the same spirit as in modern lattice-QCD calculations.

In the present short review, we first show why we believe the $X(4260)$ enhancement, discovered by the BABAR collaboration, not to be a resonance, on the basis of the BABAR data themselves. Next, the Resonance-Spectrum Expansion (RSE) is briefly revisited, which is the non-perturbative and exactly solvable unquenched formalism we employ to describe non-exotic mesonic resonances. The RSE method is then applied to the $X(3872)$ charmonium state and the axial-vector charmed mesons $D_{1}(2420), D_{1}(2430), D_{s 1}(2536)$, and $D_{s 1}(2460)$. Finally, as a surprising additional result, we present indications of a so far unobserved very light scalar boson, from analysing published BABAR data.

\section{Non-resonant charmonium enhancement $X(4260)$}

In 2005, the BABAR Collaboration observed 88 the vector charmonium enhancement $X(4260)$ in $\pi^{+} \pi^{-} J / \psi$ data, which was later confirmed by other collaborations and also in different production processes, though not in open-charm channels [1]. The non-observation of OZI-allowed decays of the $X(4260)$, as well as its awkward mass for most potential models, led to various exotic or molecular model explanations (see Ref. 9] for some references). However, another very striking feature of the $X(4260)$ data, viz. a conspicuous dip precisely at the mass of the well-established $\psi(4415)$ [1] resonance, is usually ignored. Moreover, none of the other known $c \bar{c}$ resonances show up in the BABAR data [8]. Nevertheless, this seemingly inexplicable behaviour of the $X(4260) \rightarrow \pi^{+} \pi^{-} J / \psi$ data can be understood if one assumes that they are depleted by competing yet not searched for open-charm decays, which effect should be most pronounced at the positions of true resonances. Such a depletion makes sense, as decays into open-charm mesons are OZI-allowed and therefore should be strongly dominant.

Reference [9] elaborated on this idea, by assuming a very broad, non-resonant structure centred on the $X(4260)$, resulting from an $f_{0}(600)$ (and possibly $f_{0}(980)$ ) peripheral emission in an OZI-forbidden production process like $e^{+} e^{-} \rightarrow c \bar{c} \rightarrow\left(f_{0}(600) \rightarrow \pi^{+} \pi^{-}\right) J / \psi$. The competing open-charm thresholds $D \bar{D}, D \bar{D}^{*}, D^{*} \bar{D}^{*}, D_{s} \bar{D}_{s}, D_{s} \bar{D}_{s}^{*}, D_{s}^{*} \bar{D}_{s}^{*}$, and $\Lambda_{c} \Lambda_{c}$, as well as the $c \bar{c}$ resonances $\psi(4040), \psi(4160), \psi(4415)$, $\psi(3 D)$, then deplete this structure, resulting in the data pattern observed by BABAR, including the $X(4260)$ enhancement, as depicted in Fig. 1. Note that the $\psi(3 D)$ state, at about $4.53 \mathrm{GeV}$, is not 


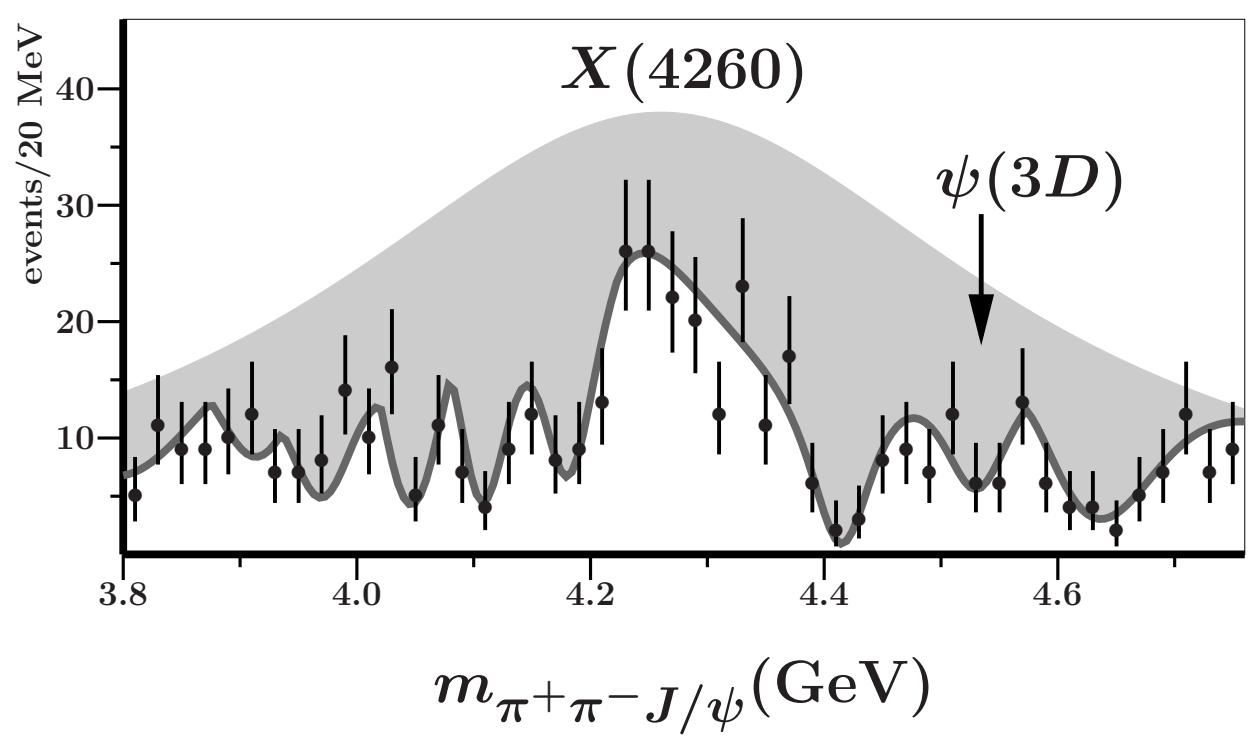

Figure 1: Depletion of very broad structure (shaded area) by $c \bar{c}$ resonances and open-charm thresholds, resulting in solid curve. Data are from Ref. [8]; see Ref. [9] for further details.

yet listed in the PDG tables [1, but its existence is supported by other data as well (see Ref. [9] and references therein, also for more details on the data parametrisation employed in Fig. (1).

\section{Resonance-Spectrum Expansion}

For the description of meson-meson scattering in non-exotic channels and resonances therein, the Resonance-Spectrum Expansion [10, 11] is a simple though powerful and only mildly model-dependent formalism. It is based on the idea that $s$-channel exchanges strongly dominate such scattering processes, giving rise to an effective meson-meson interaction that is separable in the relative momenta, as can be seen from Fig. 2. However, contrary to other effective approaches in which sometimes $s$-channel seeds
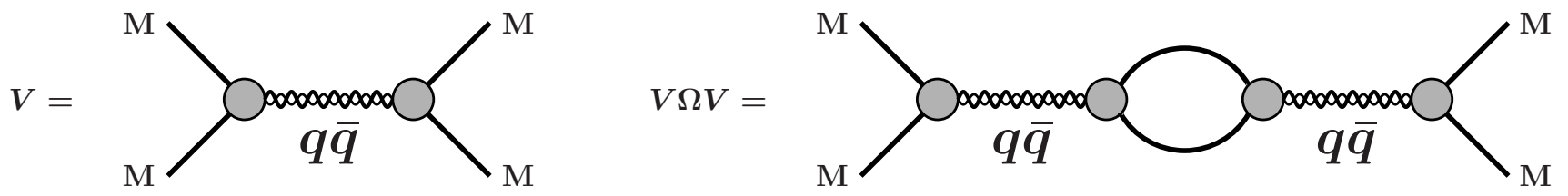

Figure 2: Left: Effective meson-meson Born diagram. Right: corresponding one-loop diagram.

are introduced, our intermediate states are described microscopically by a complete spectrum of bare quark-antiquark systems, thus resembling Regge propagators [10] (see the wiggly lines in Fig. 22). The transitions between the $q \bar{q}$ and meson-meson systems are supposed to take place via ${ }^{3} P_{0}$ pair creation or annihilation, modelled via a spherical delta function in coordinate space, which mimics string breaking at a well-defined separation. The resulting vertex functions, represented by the blobs in the Born and one-loop diagrams in Fig. 2, are spherical Bessel functions in momentum space. Owing to the separability of the effective interaction, the corresponding $T$-matrix can be solved in closed form. In the general case of $N_{q \bar{q}} q \bar{q}$ channels coupled to $N_{M M}$ meson-meson channels, the interaction reads

$$
V_{i j}^{\left(L_{i}, L_{j}\right)}\left(p_{i}, p_{j}^{\prime} ; E\right)=\lambda^{2} r_{0} j_{L_{i}}^{i}\left(p_{i} r_{0}\right) j_{L_{j}}^{j}\left(p_{j}^{\prime} r_{0}\right) \sum_{\alpha=1}^{N_{q \bar{q}}} \sum_{n=0}^{\infty} \frac{g_{i}^{(\alpha)}(n) g_{j}^{(\alpha)}(n)}{E-E_{n}^{(\alpha)}} \equiv \mathcal{R}_{i j}(E) j_{L_{i}}^{i}\left(p_{i} r_{0}\right) j_{L_{j}}^{j}\left(p_{j}^{\prime} r_{0}\right),
$$


leading to the fully off-energy-shell $T$-matrix

$$
T_{i j}^{\left(L_{i}, L_{j}\right)}\left(p_{i}, p_{j}^{\prime} ; E\right)=-2 \lambda^{2} r_{0} \sqrt{\mu_{i} p_{i} \mu_{j}^{\prime} p_{j}^{\prime}} j_{L_{i}}^{i}\left(p_{i} r_{0}\right) \sum_{m=1}^{N} \mathcal{R}_{i m}(E)\left\{[\mathbb{1}-\Omega \mathcal{R}]^{-1}\right\}_{m j} j_{L_{j}}^{j}\left(p_{j}^{\prime} r_{0}\right),
$$

with the loop function

$$
\Omega=-2 i \lambda^{2} r_{0} \operatorname{diag}\left(j_{L_{n}}^{n}\left(k_{n} r_{0}\right) h_{L_{n}}^{(1) n}\left(k_{n} r_{0}\right)\right)
$$

Here, $\lambda$ is an overall coupling, $r_{0}$ is the average distance for decay via ${ }^{3} P_{0}$ quark-pair creation, $E_{n}^{(\alpha)}$ is the discrete energy of the $n$-th recurrence in $q \bar{q}$ channel $\alpha, g_{i}^{(\alpha)}$ is the corresponding coupling to the $i$ th meson-meson channel, $\mu_{i}$ the reduced mass for this channel, $p_{i}$ the off-shell relative momentum, $L_{i}$ the orbital angular momentum, and $j_{L_{i}}^{i}$ and $h_{L_{j}}^{(1) j}\left(k_{j} r_{0}\right)$ the spherical Bessel function and Hankel function of the first kind, respectively. Note that $\mu_{i}, p_{i}$, and the on-energy-shell relative momentum $k_{i}$ are defined relativistically. The $S$-matrix is then given by $S_{i j}^{\left(L_{i}, L_{j}\right)}(E)=1+2 i T_{i j}^{\left(L_{i}, L_{j}\right)}\left(k_{i}, k_{j} ; E\right)$.

\section{$4 X(3872)$ as a unitarised $1^{++} c \bar{c}$ state}

The $X(3872)$ charmonium-like state was discovered in 2003 by the Belle Collaboration [12], as a $\pi^{+} \pi^{-} J / \psi$ enhancement in the decay $B^{ \pm} \rightarrow K^{ \pm} \pi^{+} \pi^{-} J / \psi$. In subsequent years, it was confirmed in other experiments, with the additional hadronic decay modes $\rho^{0} J / \psi, \omega J / \psi, D^{0} \bar{D}^{0} \pi^{0}$, and $D^{0} \bar{D}^{* 0}$ being seen as well [1. Although the $X(3872)$ decay modes are perfectly compatible with a $2{ }^{3} P_{1}$ or $1{ }^{1} D_{2}$ assignment [1], the former $c \bar{c}$ state is usually thought to be roughly $100 \mathrm{MeV}$ heavier, whereas the latter is mostly predicted to be $30-80 \mathrm{MeV}$ lighter. Also because of the $X(3872)$ 's remarkable closeness to the $D^{0} D^{* 0}$ threshold, many authors have proposed exotic or molecular configurations (see Ref. [13] for some references).

However, in Ref. 13] we showed that the $X(3872)$ can be very well described as the first radial excitation of the $1^{3} P_{1} \chi_{c 1}(3511)$, i.e., with quantum numbers $J^{P C}=1^{++}$. By coupling the relevant open and closed open-charm channels to a bare $2^{3} P_{1} c \bar{c}$ state, its mass gets shifted downwards by roughly $100 \mathrm{MeV}$, while the corresponding pole settles almost on top of or slightly below the $D^{0} D^{* 0}$ threshold. In Fig. 3, left-hand plot, we display the $X(3872)$ pole trajectory as a function of the overall
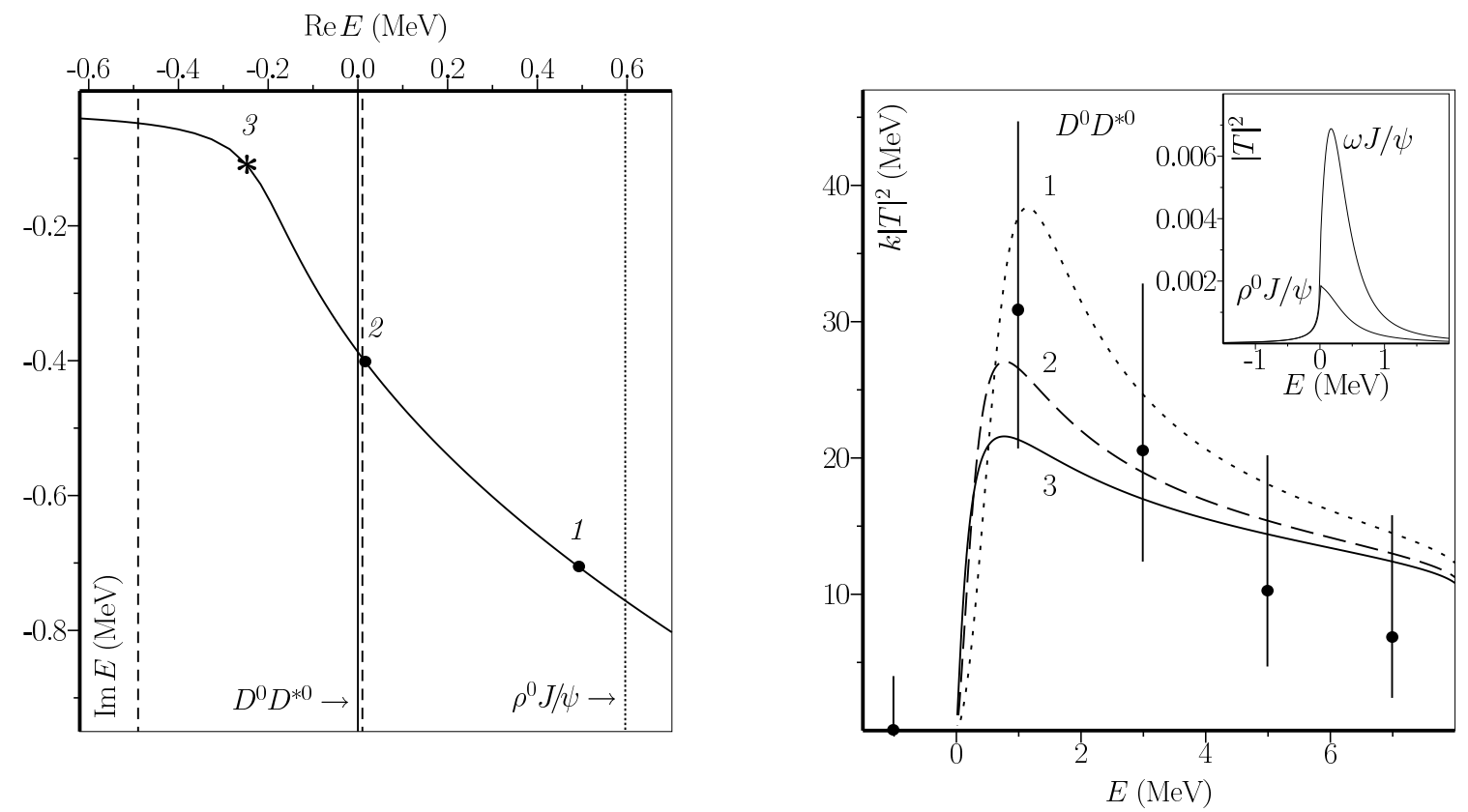

Figure 3: Left: $X(3872)$ pole trajectory as a function of $\lambda$; dashed lines delimit allowed [1] pole range. Right: $D^{0} D^{* 0}$ amplitude with data [12]; inset: relative comparison of $\rho^{0} \mathrm{~J} / \psi$ and $\omega \mathrm{J} / \psi$ amplitudes. 
coupling $\lambda$ in the vicinity of the $D^{0} D^{* 0}$ threshold, for values of $\lambda$ close to 3 . The two bullets and the star correspond to the pole positions 1,2,3 for which the $D^{0} D^{* 0}$ amplitude is depicted in the right-hand plot. All three cases are compatible with the data [12], though pole no. 3 agrees best with the PDG value of $(3871.57 \pm 0.25) \mathrm{MeV}$ [1] for the $X(3872)$ mass. The inset in the latter plot compares the amplitudes in the OZI-forbidden channels $\omega J / \psi$ and $\rho^{0} J / \psi$, which are coupled to the $X(3872)$ system, too. Although their influence on the pole positions is almost negligible, owing to their small couplings, they must nevertheless be included for a realistic description of the $\pi^{+} \pi^{-} \pi^{0} J / \psi$ and $\pi^{+} \pi^{-} J / \psi$ data, respectively. What the inset shows is that the $\omega J / \psi$ and $\rho^{0} J / \psi$ amplitudes are of comparable size, at least at the energy of the pole (no. 2 in this case), despite the fact that the $\omega \mathrm{J} / \psi$ coupling is much larger than that of the isospin-breaking $\rho^{0} \mathrm{~J} / \psi$ channel. Note that the latter channels are effectively smeared out in order to account for the widths of the $\omega$ and especially the very broad $\rho$. This is done using a novel unitarisation procedure for an $S$-matrix with complex masses in the asymptotic states [13]. Thus, the $\rho^{0} J / \psi$ decay mode gets enhanced, such that $\Gamma(\omega J / \psi) / \Gamma\left(\rho^{0} J / \psi\right) \sim 1$, in agreement with data [1].

\section{$5 \quad$ Understanding the axial-vector charmed mesons}

One of the major puzzles in open-charm spectroscopy is the seemingly odd pattern of masses and widths of the axial-vector (AV) charmed mesons $D_{1}(2420), D_{1}(2430), D_{s 1}(2536)$, and $D_{s 1}(2460)$ [1. In the first place, the two $c \bar{q}(q=u, d)$ states are almost degenerate in mass, while there is a $76 \mathrm{MeV}$ mass splitting between the two $c \bar{s}$ AV mesons. Secondly, the $D_{1}(2420)$ and $D_{s 1}(2536)$ resonances are much narrower than what one would naively expect from their OZI-allowed $S$-wave decay modes $D^{*} \pi$ and $D^{*} K$, respectively. Finally, both $c \bar{s}$ states are very narrow $(0-3 \mathrm{MeV})$, whereas the $D_{1}(2430)$ is very broad ( $\sim 400 \mathrm{MeV})$ and the $D_{1}(2420)$ has a modest width $(20-25 \mathrm{MeV})$. Standard quark models as well as chiral-Lagrangian approaches to heavy-light mesons completely fail to predict such a pattern [14].

In Ref. [14, we tackled the AV charmed mesons by coupling bare $c \bar{q}$ and $c \bar{s}$ states, each with a ${ }^{3} P_{1}$ and a ${ }^{1} P_{1}$ component, to the relevant OZI-allowed meson-meson channels, most importantly $D^{*} \pi$ for $c \bar{q}$ and $D^{*} K$ for $c \bar{s}$. Notice that charmed mesons have no definite $C$-parity, so that both ${ }^{3} P_{1}\left(J^{P C}=1^{++}\right)$ and ${ }^{1} P_{1}\left(J^{P C}=1^{+-}\right)$contribute to the wave function. In Fig. 4, we show how the bare $c \bar{q}$ and $c \bar{s}$ states
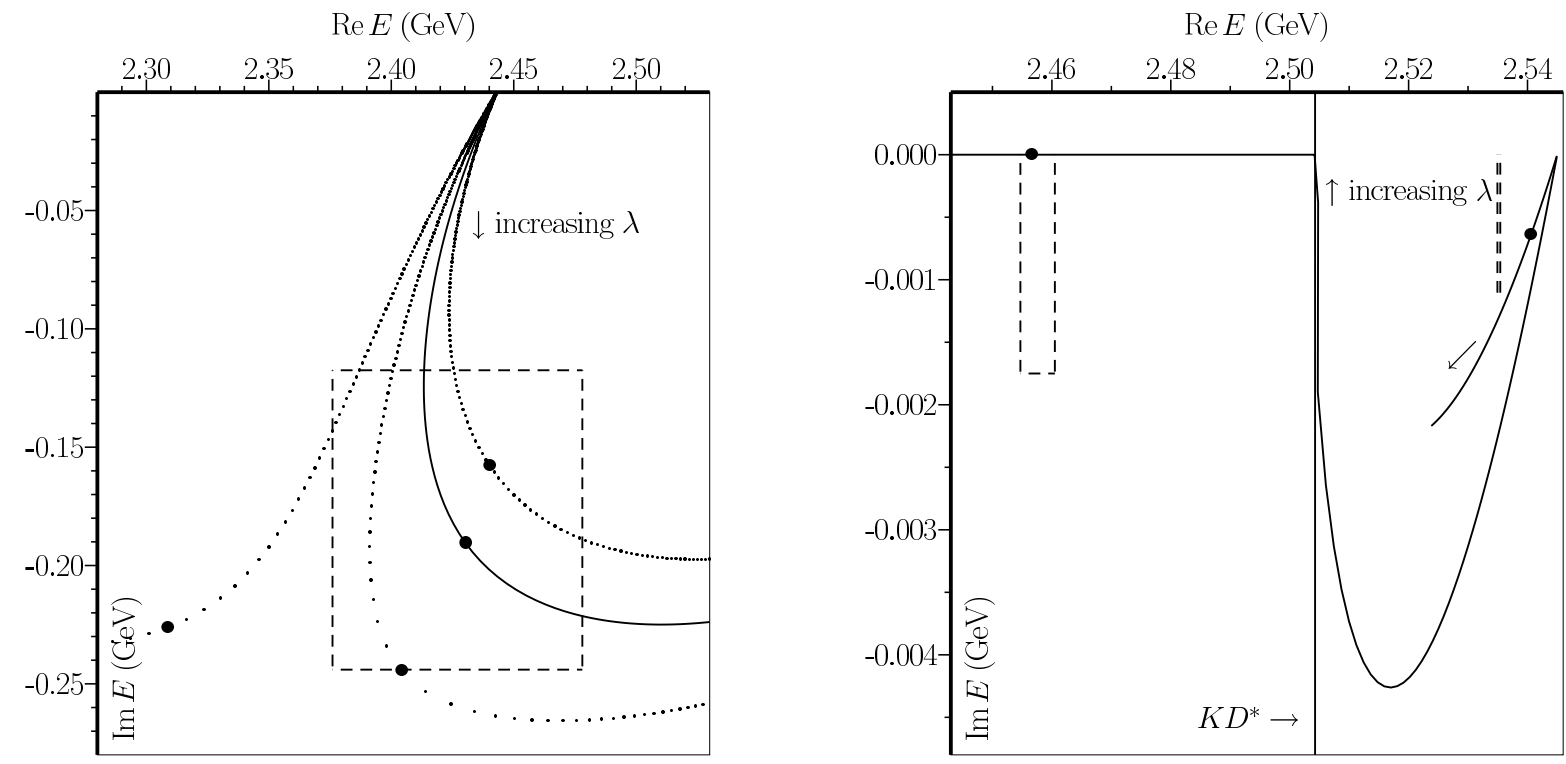

Figure 4: Left: pole trajectories of $D_{1}(2430)$ as a function of $\lambda$, for $r_{0}=3.2-3.5 \mathrm{GeV}^{-1}$; right: trajectories of $D_{s 1}(2536)$ and $D_{s 1}(2460)$, as a function of $\lambda$, for $r_{0}=3.12 \mathrm{GeV}^{-1}$. Left and right: dashed box, rectangle, and strip delimit experimental [1] $D_{1}(2430), D_{s 1}(2460)$, resp. $D_{s 1}(2536)$ pole. Also see text. 
at 2443 and $2545 \mathrm{MeV}$ [14], respectively, get shifted and split up because of the coupled meson-meson channels. However, in either case, one pole moves very little, thus behaving as a quasi-bound state in the continuum [14], whereas the other shifts a lot. Note that the relatively narrow $D_{1}(2420)$, with pole at $(2439-i 3.5) \mathrm{MeV}$, is not shown in Fig. 4, for clarity. Also, the bullets correspond to the optimum coupling values $\lambda=1.30$ (1.19) for $c \bar{q}(c \bar{s})$. As a result, the $D_{1}(2430)$ becomes very broad, but with practically the same central mass as the $D_{1}(2420)$. On the other hand, the $D_{s 1}(2460)$ pole does move to lower energies, even below the $D^{*} K$ threshold, in agreement with experiment. Overall, four masses and four widths are quite well reproduced with only two parameters, which moreover have little freedom. Moreover, the mixing angle of about $35^{\circ}$ between the ${ }^{3} P_{1}$ and ${ }^{1} P_{1}$ components, needed to describe the data, is generated here fully dynamically and not put in by hand. For further details, see Ref. [14].

\section{Surprise in BABAR data: a very light scalar of $38 \mathrm{MeV}$ ?}

Observation of the $\Upsilon\left(2^{3} D_{1}\right)$ [15] and seeing indications of the $\Upsilon\left(1^{3} D_{1}\right)$ [15] in published [16] BABAR data stimulated further analysis of these data. Surprisingly, evidence was found [17] for the existence of a very light scalar boson of strong interactions, with a mass of about $38 \mathrm{MeV}$. This was based on clear and structured excess signals in invariant-mass projections of $e^{+} e^{-}$and $\mu^{+} \mu^{-}$pairs for the reactions $e^{+} e^{-} \rightarrow \pi^{+} \pi^{-} \Upsilon\left(1,2{ }^{3} S_{1}\right) \rightarrow \pi^{+} \pi^{-} e^{+} e^{-}$(and $\rightarrow \pi^{+} \pi^{-} \mu^{+} \mu^{-}$). In fact, similar excess signals were observed, though not explained, in Ref. [18, for the process $\Upsilon(3 S) \rightarrow \pi^{+} \pi^{-} \Upsilon(1 S) \rightarrow \pi^{+} \pi^{-} \mu^{+} \mu^{-}$, as depicted here in Fig. 5, upper plot. In the lower plot, we show very similar signals, with the same
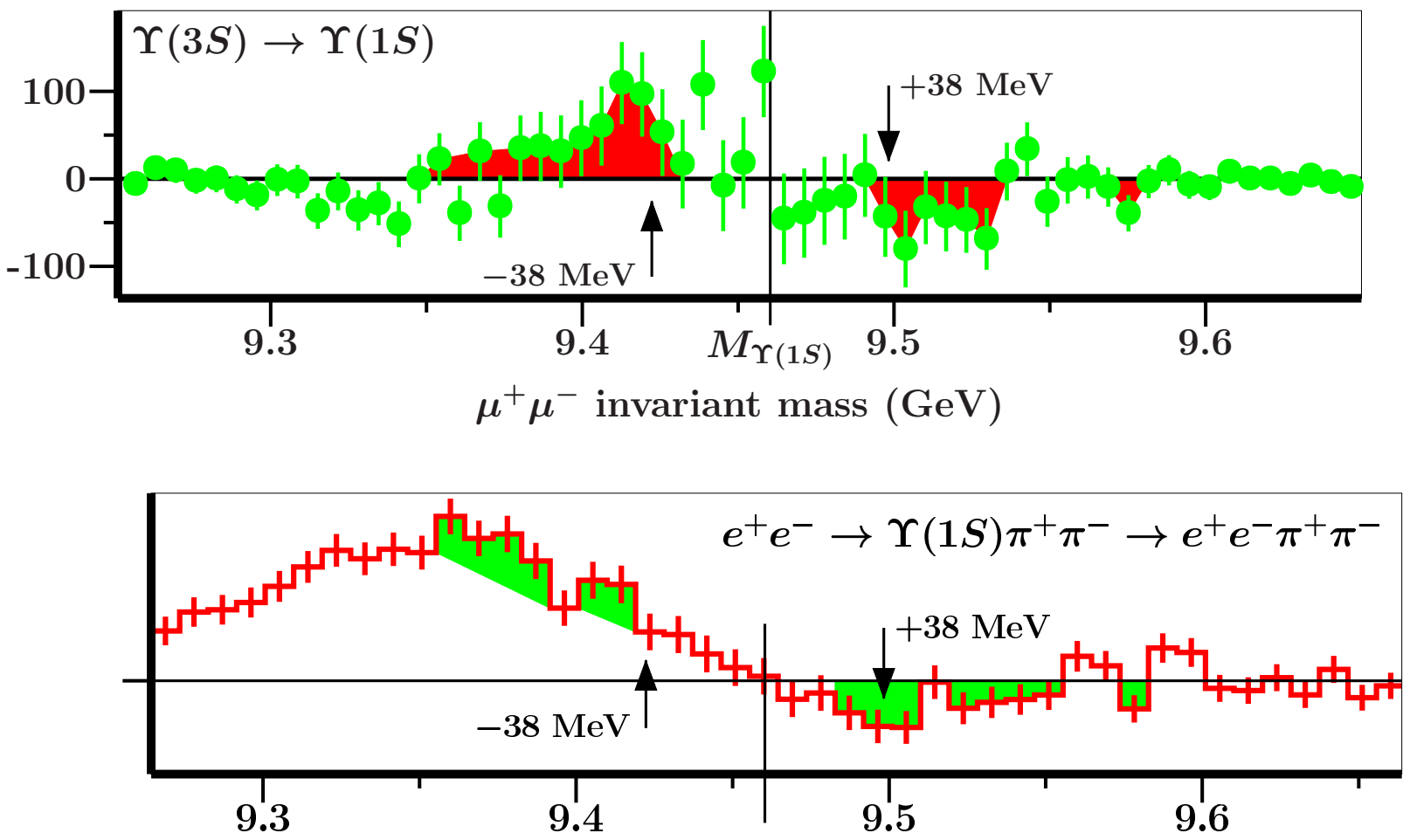

Figure 5: Top: event distribution of the excess signal [18] in the invariant $\mu^{+} \mu^{-}$mass for the reaction $\Upsilon\left(3{ }^{3} S_{1}\right) \rightarrow \pi^{+} \pi^{-} \Upsilon\left(1{ }^{3} S_{1}\right) \rightarrow \pi^{+} \pi^{-} \mu^{+} \mu^{-}$, using bins of $6.5 \mathrm{MeV}$. Bottom: excess signals in invariant $e^{+} e^{-}$mass distributions for the reaction $e^{+} e^{-} \rightarrow \pi^{+} \pi^{-} \Upsilon\left(1{ }^{3} S_{1}\right) \rightarrow \pi^{+} \pi^{-} e^{+} e^{-}$, using all available data [16] and bins of $9 \mathrm{MeV}$; statistical errors are shown by vertical bars.

structure, in $e^{+} e^{-} \rightarrow \pi^{+} \pi^{-} \Upsilon(1 S) \rightarrow \pi^{+} \pi^{-} e^{+} e^{-}$data [16], which clearly suggest emission of a light quantum. For discussion, theoretical justifications, and more experimental evidence supporting a very light scalar for strong interactions, see Ref. [17]. 


\section{Conclusions}

In the foregoing we have shown, at the hand of several examples of puzzling mesonic structures, that genuine meson spectroscopy is much more involved than just hunting after the biggest bump in data and trying to reproduce its central mass with some confining potential. In the case of true resonances, like the $X(3872)$ and the AV charmed mesons $D_{1}(2420), D_{1}(2430), D_{s 1}(2536)$, and $D_{s 1}(2460)$, large and non-linear effects are found from unitarisation, which makes a reliable determination of their masses and widths a complicated task. For non-resonant enhancements like the $X(4260)$ — in our view a very careful analysis of the available data is required, as well as accounting for competing channels and existing resonances in the same energy region. We believe that the RSE, both for scattering, as described in Sec. 3, and for production processes [11], is a very powerful yet simple-to-use formalism, which can be adapted to any quark potential model.

Unquenching is becoming more and more mandatory in quark models, just like in lattice QCD. To think that its effects can be neglected in spectroscopy, or mimicked by a global redefinition of quark masses, is an illusion.

\section{Acknowledgements}

Two of us (EvB, GR) thank J. Segovia for collaboration on the X(4260) (Sec. 2). This work was supported in part by the Fundação para a Ciência e a Tecnologia of the Ministério da Ciência, Tecnologia e Ensino Superior of Portugal, under contract CERN/FP/116333/2010 and grant SFA-2-91/CFIF.

\section{References}

[1] K. Nakamura et al. [Particle Data Group], J. Phys. G 37 (2010) 075021 and 2011 online update

[2] E. van Beveren and G. Rupp, PoS HQL2010 (2010) 003 [arXiv:1011.2360 [hep-ph]]

[3] I. Adachi et al. [Belle Collaboration], arXiv:1105.4583 [hep-ex]

[4] D. V. Bugg, Europhys. Lett. 96 (2011) 11002 [arXiv:1105.5492 [hep-ph]]

[5] I. V. Danilkin, V. D. Orlovsky, and Yu. A. Simonov, arXiv:1106.1552 [hep-ph]

[6] E. van Beveren, T. A. Rijken, K. Metzger, C. Dullemond, G. Rupp, and J. E. Ribeiro, Z. Phys. C 30 (1986) 615 arXiv:0710.4067 [hep-ph]]

[7] E. van Beveren, D. V. Bugg, F. Kleefeld, and G. Rupp, Phys. Lett. B 641 (2006) 265 arXiv:hep-ph/0606022

[8] B. Aubert et al. [BABAR Collaboration], Phys. Rev. Lett. 95 (2005) 142001 arXiv:hep-ex/0506081

[9] E. van Beveren, G. Rupp, and J. Segovia, Phys. Rev. Lett. 105 (2011) 102001 arXiv:1005.1010 [hep-ph]]

[10] E. van Beveren and G. Rupp, Ann. Phys. 324 (2009) 1620 arXiv:0809.1149 [hep-ph]]

[11] E. van Beveren and G. Rupp, Ann. Phys. 323 (2008) 1215 arXiv:0706.4119 [hep-ph]]

[12] S. K. Choi et al. [Belle Collaboration], Phys. Rev. Lett. 91 (2003) 262001 arXiv:hep-ex/0309032

[13] S. Coito, G. Rupp, and E. van Beveren, Eur. Phys. J. C. 71 (2011) 1762 arXiv:1008.5100 [hep-ph]

[14] S. Coito, G. Rupp and E. van Beveren, Phys. Rev. D 84 (2011) 094020 arXiv:1106.2760 [hep-ph]

[15] E. van Beveren and G. Rupp, arXiv:1009.4097 [hep-ph]

[16] B. Aubert et al. [BABAR Collaboration], Phys. Rev. D 78 (2008) 112002 [arXiv:0807.2014 [hep-ex]]

[17] E. van Beveren and G. Rupp, arXiv:1102.1863 [hep-ph]

[18] E. Guido [BABAR Collaboration], arXiv:0910.0423 [hep-ex] 